\title{
A structural risk-neutral model of electricity prices*
}

\author{
René Aïd ${ }^{\dagger} \quad$ Luciano Campi ${ }^{\ddagger} \quad$ Adrien Nguyen Huu ${ }^{\S} \quad$ Nizar Touzi ${ }^{\Uparrow}$
}

June 2009

\begin{abstract}
The objective of this paper is to present a model for electricity spot prices and the corresponding forward contracts, which relies on the underlying market of fuels, thus avoiding the electricity nonstorability restriction. The structural aspect of our model comes from the fact that the electricity spot prices depend on the dynamics of the electricity demand at the maturity $T$, and on the random available capacity of each production means. Our model explains, in a stylized fact, how the prices of different fuels together with the demand combine to produce electricity prices. This modeling methodology allows one to transfer to electricity prices the risk-neutral probabilities of the market of fuels and under the hypothesis of independence between demand and outages on one hand, and prices of fuels on the other hand, it provides a regression-type relation between electricity forward prices and forward prices of fuels. Moreover, the model produces, by nature, the well-known peaks observed on electricity market data. In our model, spikes occur when the producer has to switch from one technology to the lowest cost available one. Numerical tests performed on a very crude approximation of the French electricity market using only two fuels (gas and oil) provide an illustration of the potential interest of this model.
\end{abstract}

Keywords: energy markets; electricity prices; fuel prices; risk-neutral probability; no-arbitrage pricing; forward contract.

JEL Classification: D41; G13. AMS Classification (2000): 91B24; 91B26.

\section{Introduction}

In security markets, the following relationship between spot and forward prices of a given security holds:

$$
F(t, T)=S_{t} e^{r(T-t)}, \quad t \leq T .
$$

As usual, $T$ is the maturity of the forward contract, $S_{t}$ is the spot price at $t$ and $r$ is the interest rate which is assumed constant for simplicity. We also assume no dividends. The no-arbitrage arguments usually used to prove such an equality lie heavily upon the fact that securities are storable at zero cost. For storable commodities (oil, soybeans, silver...), the former relation has been extended by including storage costs and and an unobservable variable called convenience yield (see Schwartz [23], [22], and Geman [17], sec. 3.7). But, when one considers electricity markets (see Burger et al. [9] or Geman and Roncoroni [18] for an exhaustive description), such a property does not hold anymore: Once purchased, the electricity has to be consumed, so that the above relation does not make sense. This fact is very well documented in electricity market literature (see, e.g., Clewlow \& Strickland [12]) but has not prevented the development of many

\footnotetext{
*The authors thank the "Chair Finance and Sustainable Development" sponsored by EDF and Calyon for their support.

${ }^{\dagger}$ EDF R\&D and FiME, Laboratoire de Finance des Marchés d'Energies.

${ }^{\ddagger}$ CEREMADE, University Paris-Dauphine \& FiME, Laboratoire de Finance des Marchés d’Energies. campi@ceremade.dauphine.fr

$\S$ EDF R\&D and CEREMADE University Paris-Dauphine.

ฯ Centre de Mathématiques Appliquées, Ecole Polytechnique Paris.
} 
electricity spot price models following the Black \& Scholes framework [5, 7, 4, 6, 10, 11, 15] (see Benth [4] for a survey of the literature).

Nevertheless, the non-storability of electricity is not enough to claim that no relation holds between spot and forward prices and that no arbitrage constraint affects the term structure of electricity prices, except the constraints coming from overlapping forward contracts. Indeed, one could argue that even if electricity can not be stored, the fuels that are used to produce electricity can. To see that this observation leads to constraints on the term structure of electricity prices, let us consider a fictitious economy in which power is produced by a single technology - coal thermal units with the same degree of efficiency - and that the electricity spot market is competitive. Then, the electricity price should satisfy the following relation :

$$
F_{e}(t, T)=q_{c} F_{c}(t, T), \quad t \leq T,
$$

where the subscript $e$ stands for electricity, $c$ stands for coal, and $q_{c}$ denotes the heat rate. If there is $t<T$ such that $F_{e}(t, T)>q_{c} F_{c}(t, T)$, then one can at time $t$ :

- Sell a forward on electricity at $F_{e}(t, T)$ and buy $q_{c}$ coal forward at $F_{c}(t, T)$

and, at time $T$ :

- Sell $q_{c}$ coal at $S_{c}(T)$, buy electricity at $S_{e}(T)=q_{c} S_{c}(T)$.

One can check that this strategy is indeed an arbitrage. Moreover, the opposite relation can be obtained in a similar way. Here, in this fictitious economy, the important feature is not that electricity can be produced by coal, but that the relation between spot prices of coal and electricity is known. Furthermore, it extends directly to forward prices.

In real economies, similar no-arbitrage relations between electricity and fuel prices can not be identified so easily. The reason for this is that electricity can be produced out of many technologies with many different efficiency levels: Coal plants more or less ancient, fuel plants, nuclear plants, hydro, solar and windfarms, and so on. Generally, the electricity spot price is considered to be the day-ahead hourly market price. At that time horizon, any producer will perform an ordering of its production means on the basis of their production costs. This process refers to a unit commitment problem and one can find a huge literature on this optimization problem in power systems literature (see, e.g., Batut and Renaud [3] and Dentcheva et al. [14]). Depending on the market prices of fuels and on the state of the power system (demand, outages, inflows, wind and so forth), this ordering may vary through time. Hence, when the forward contract is being signed, the ordering at the contract maturity is not known.

The objective of this paper is to build a model for electricity spot prices and the corresponding forward contracts, which relies on the underlying markets of fuels, thus avoiding the non-storability restriction. The structural aspect of our model comes from the fact that the electricity spot prices depend on the dynamics of the electricity demand at the maturity $T$, and on the random available capacity of each production means. Our model allows one to explain, in a stylized fact, how the prices of different fuels together with the demand combine to produce electricity prices. This modeling methodology allows us to transfer to electricity prices the risk-neutral probabilities of the market of fuels, under a certain independence hypothesis (see Assumption 2.2). Moreover, the model produces, by nature, the well-known peaks observed on electricity market data. In our model, spikes occur when the producer has to switch from one technology to the lowest cost available one. Moreover, the dynamics of the demand explain this switching process. Then, one easily understands that the spikes result from a high level of the demand which forces the producer to use a more expensive technology.

Our model is close to Barlow's [2], since the electricity spot price is defined as an equilibrium between demand and production. But, in our model, the stack curve is described by the different available capacities and not a single parametrized curve. Moreover, this model shares some ideas with Fleten and Lemming forward curve reconstruction method [16]. But, whereas the authors methodology relies on an external structural model provided by the SINTEF, our methodology does not require such inputs.

The article is structured in the following way: Section 2 is devoted to the description of our model; Section 3 describes the relation between future prices of electricity and fuels; Section 4 presents the model 
in the case of only two fuels; Section 5 presents numerical results showing the potential of the model on the two technologies case of the preceeding section; finally, Section 6 provides some future research perspectives.

\section{The Model}

Let $(\Omega, \mathcal{F}, \mathbb{P})$ be a probability space sufficiently rich to support all the processes we will introduce throughout this paper. Let $\left(W^{0}, W\right)$ be an $(n+1)$-dimensional standard Wiener process with $W=\left(W^{1}, \ldots, W^{n}\right)$, $n \geq 1$. In the sequel, we will distinguish between the filtration $\mathcal{F}^{0}=\left(\mathcal{F}_{t}^{0}\right)$ generated by $W^{0}$ and the filtration $\mathcal{F}^{W}=\left(\mathcal{F}_{t}^{W}\right)$ generated by the $n$-dimensional Wiener process $W=\left(W^{1}, \ldots, W^{n}\right)$.

Commodities market. We consider a market where agents can trade $n \geq 1$ commodities and purchase electricity. We consider only commodities that can be used to produce electricity. With a slight abuse of language, we will always identify in this paper any given production technology with the corresponding commodity (also called fuel) used. For $i=1, \ldots n, S_{t}^{i}$ denotes the price of the quantity of commodity $i$ necessary to produce $1 \mathrm{KWh}$ of electricity and is assumed to follow the following SDE:

$$
d S_{t}^{i}=S_{t}^{i}\left(\mu_{t}^{i} d t+\sum_{j=1}^{n} \sigma_{t}^{i j} d W_{t}^{j}\right), \quad t \geq 0,
$$

where $\mu^{i}$ and $\sigma^{i j}$ are $\mathcal{F}^{W}$-adapted processes suitably integrable (see Assumption 2.1).

We also assume that the market contains a riskless asset with price process

$$
S_{t}^{0}=e^{\int_{0}^{t} r_{u} d u}, t \geq 0
$$

where the instantaneous interest rate $\left(r_{t}\right)_{t \geq 0}$ is an $\mathcal{F}^{W}$-adapted non-negative process such that $\int_{0}^{t} r_{u} d u$ is finite a.s. for every $t \geq 0$. As a consequence, $\left(r_{t}\right)$ is independent of the Brownian motion $W^{0}$. We will frequently use the notation $\tilde{X}_{t}:=X_{t} / S_{t}^{0}$ for any process $\left(X_{t}\right)$. We make the following standard assumption (see, e.g. Karatzas [20], Section 5.6).

Assumption 2.1 The volatility matrix $\sigma_{t}=\left(\sigma_{t}^{i j}\right)_{1 \leq i, j \leq n}$ is invertible and both matrices $\sigma$ and $\sigma^{-1}$ are bounded uniformly on $\left[0, T^{*}\right] \times \Omega$. Finally, let $\theta$ denote the market price of risk, i.e.

$$
\theta_{t}:=\sigma_{t}^{-1}\left[\mu_{t}-r_{t} \mathbf{1}_{n}\right], t \geq 0,
$$

where $\mathbf{1}_{n}$ is the $n$-dimensional vector with all unit entries. We assume that such a process $\theta$ satisfies the so-called Novikov condition

$$
\mathbb{E}\left[\exp \left\{\frac{1}{2} \int_{0}^{T^{*}}\left\|\theta_{t}\right\|^{2} d t\right\}\right]<\infty \text { a.s. }
$$

Remark 1 Imposing the Novikov condition on the commodities market price of risk ensures that the minimal martingale measure we will use for pricing in Section 3 is well defined. The reader is referred to Section 5.6 in Karatzas's book [20].

Market demand for electricity. We model the electricity market demand by a real-valued continuous process $D=\left(D_{t}\right)_{t \geq 0}$ adapted to the filtration $\mathcal{F}^{0}=\left(\mathcal{F}_{t}^{0}\right)$ generated by the Brownian motion $W^{0}$. Observe that, under our assumptions, the processes $S^{i}(i=0, \ldots, n)$ are independent under $\mathbb{P}$ of the demand process $D$. To be more precise, the process $D$ models the whole electricity demand of a given geographical area (e.g. U.K., Switzerland, Italy and so on). In this respect, it must be strictly positive. Nevertheless, in Section 5, where the empirical analysis is performed, it is more convenient to use a residual demand to reduce the number of possible technologies. A residual demand is the whole demand less the production of some generation assets (e.g. nuclear power, run of the river hydrolic plants, wind farms). It is clear that the residual demand can be negative. 
Electricity spot prices. We denote by $P_{t}$ the electricity spot price at time $t$. At any time $t$, the electricity producer can choose among the $n$ commodities which is the most convenient to produce electricity at that particular moment and the electricity spot price will be proportional to the spot price of the chosen commodity. We recall that the proportionality factor is already included in the definition of each $S^{i}$ so that, if at time $t$ the producer chooses commodity $i$ then $P_{t}=S_{t}^{i}, 1 \leq i \leq n$.

How does the electricity producer choose the most convenient commodity to use? For each $i=1, \ldots, n$, we denote $\Delta_{t}^{i}>0$ the given capacity of the $i$-th technology for electricity production at time $t .\left(\Delta_{t}^{i}\right)$ is a stochastic process defined on $(\Omega, \mathcal{F}, \mathbb{P})$ and assumed independent of $\left(W^{0}, W\right)$. We denote $\mathcal{F}^{\Delta}=\left(\mathcal{F}_{t}^{\Delta}\right)$ its filtration. Moreover, we assume that each $\Delta_{t}^{i}$ takes values in $\left[m_{i}, M_{i}\right]$ where $0 \leq m_{i}<M_{i}$ are the minimal and the maximal capacity of $i$-th technology, both values being known to the producer. In reality, the producer has to fill capacity constraints, so he faces demand variability, security conditions and failures risk. Thus, if one wants to consider capacity management and partial technology failures in the model, the production capacity has to be modelled as a stochastic process.

For every given $(t, \omega)$, the producer performs an ordering of the commodities from the cheapest to the most expensive. The ordered prices of commodities are denoted by

$$
S_{t}^{(1)}(\omega) \leq \cdots \leq S_{t}^{(n)}(\omega)
$$

This order induces a permutation over the index set $\{1, \ldots, n\}$ denoted by $\pi_{t}=\left\{\pi_{t}(1), \ldots, \pi_{t}(n)\right\}$. Notice that $\pi_{t}$ defined an $\mathcal{F}^{W}$-adapted stochastic process, and we follow the usual probabilistic notation omitting its dependence on $\omega$.

Given a commodities order $\pi_{t}$ at time $t$, we set

$$
I_{k}^{\pi_{t}}(t):=\left[\sum_{i=1}^{k-1} \Delta_{t}^{\pi_{t}(i)}, \sum_{i=1}^{k} \Delta_{t}^{\pi_{t}(i)}\right), \quad 1 \leq k \leq n,
$$

with the convention $\sum_{i=1}^{0} \equiv 0$.

For the sake of simplicity, we will assume from now on that the electricity market is competitive and we will not take into account the short term constraints on generation assets as well as start-up costs. Hence, the electricity spot price equals the cost of the last production unit used in the stack curve (marginal unit). Thus, if the market demand at time $t$ for electricity $D_{t}$ belongs to the interval $I_{k}^{\pi_{t}}(t)$, the last unit of electricity is produced by means of technology $\pi_{t}(k)$, when available. Otherwise, it is produced with the next one with respect to the time- $t$ order $\pi_{t}$. This translates into the following formula:

$$
P_{t}=\sum_{i=1}^{n} S_{t}^{(i)} \mathbf{1}_{\left\{D_{t} \in I_{i}^{\pi_{t}}(t)\right\}}, \quad t \geq 0 .
$$

Let $T^{*}>0$ be a given finite horizon, in the sequel we will work on the finite time interval $\left[0, T^{*}\right]$. Typically, all maturities and delivery dates of forward contracts we will consider in the sequel, will always belong to $\left[0, T^{*}\right]$.

Assumption 2.2 Let $\mathcal{F}_{t}=\mathcal{F}_{t}^{0} \vee \mathcal{F}_{t}^{W} \vee \mathcal{F}_{t}^{\Delta}, t \in\left[0, T^{*}\right]$, be the market filtration. There exists an equivalent probability measure $\mathbb{Q} \sim \mathbb{P}$ defined on $\mathcal{F}_{T^{*}}$, such that the discounted prices of commodities $\tilde{S}=\left(\tilde{S}^{1}, \ldots, \tilde{S}^{n}\right)$ (i.e. without electricity!) are local $\mathbb{Q}$-martingales with respect to $\left(\mathcal{F}_{t}\right)$.

This hypothesis is equivalent to assuming absence of arbitrage in the market of fuels (see [13]). Notice that we are not making this assumption on the electricity market, as announced in the introduction. Thanks to relation (2.2), any electricity derivative can be viewed as a basket option on fuels. Hence, Assumption 2.2 allows us to properly apply the usual risk neutral machinery to price electricity derivatives. 
The market of commodities and electricity is clearly incomplete, due to the presence of additional unhedgeable randomness source $W^{0}$ driving electricity demand $D$. Thus, in order to price derivatives on electricity we have to choose an equivalent martingale measure among infinitely many to use as a pricing measure. One possible choice is the following: Let $\mathbb{Q}=\mathbb{Q}^{\text {min }}$ denote the minimal martingale measure introduced by Föllmer and Schweizer [19], i.e.

$$
\frac{d \mathbb{Q}}{d \mathbb{P}}=\exp \left\{-\int_{0}^{T^{*}} \theta_{u} \cdot d W_{u}-\frac{1}{2} \int_{0}^{T^{*}}\left\|\theta_{u}\right\|^{2} d u\right\}
$$

where we recall that $\theta_{t}=\sigma_{t}^{-1}\left(\mu_{t}-r_{t} \mathbf{1}_{n}\right)$ is the market price of risk for the commodities market $\left(S^{1}, \ldots, S^{n}\right)$. In the previous formula as well as in the sequel of this paper $x \cdot y$ denotes the scalar product between two vectors $x, y$.

Notice that, due to Assumption 2.1, such a measure is well defined, i.e. (2.3) defines a probability measure on $\mathcal{F}_{T^{*}}$, which is equivalent to the objective measure $\mathbb{P}$.

Remark 2 Furthermore, it can be easily checked that under $\mathbb{Q}$ the laws of processes $W^{0}$ and $\Delta^{i}(1 \leq i \leq n)$ are the same as under the objective probability $\mathbb{P}$ and the independence between the filtrations $\mathcal{F}^{0}, \mathcal{F}^{\Delta}$ and $\mathcal{F}^{W}$ is preserved under $\mathbb{Q}$. This property will be very useful in the proof of Proposition 3.1.

Under such a probability $\mathbb{Q}$ the prices of commodites $S^{i}, 1 \leq i \leq n$, satisfy the SDEs

$$
d S_{t}^{i}=S_{t}^{i}\left(r_{t} d t+\sum_{j=1}^{d} \sigma_{t}^{i, j} d W_{t}^{\mathbb{Q}, j}\right), \quad S_{0}^{i}>0
$$

whose solutions are given by

$$
S_{t}^{i}=S_{0}^{i} \exp \left\{\int_{0}^{t}\left(r_{u}-\frac{1}{2}\left\|\sigma_{u}^{i}\right\|^{2}\right) d u+\int_{0}^{t} \sigma_{u}^{i} \cdot d W_{u}^{\mathbb{Q}}\right\}, \quad t \geq 0,
$$

where $W^{\mathbb{Q}}=\left(W^{\mathbb{Q}, 1}, \ldots, W^{\mathbb{Q}, d}\right)$ is an $n$-dimensional Brownian motion under $\mathbb{Q}$, and $\sigma^{i}=\left(\sigma^{i, 1}, \ldots, \sigma^{i, n}\right)$.

The measure $\mathbb{Q}$ will be used as pricing measure in the rest of the paper. We recall that in the literature, such a measure $\mathbb{Q}$ is related to locally risk minimization procedure, in the sense that, given a contingent claim $H$ with some maturity $T>0, \mathbb{E}_{\mathbb{Q}}[\widetilde{H}]$ is the minimum price allowing an agent to approximately (and locally in $L^{2}$ ) hedge the claim (see Schweizer's survey [24] for further details).

Remark 3 Notice that including storage costs $c^{i}$ and convenience yields $\delta^{i}$ changes only the drift coefficients in commodities dynamics from $r_{t}$ to $r_{t}+c_{i}-\delta_{i}$.

\section{$3 \quad$ Electricity forward prices}

We now consider a so-called forward contract on electricity with maturity $T_{1}>0$ and delivery period $\left[T_{1}, T_{2}\right]$ for $T_{1}<T_{2} \leq T^{*}$, i.e. a contract defined by the payoff

$$
\left(T_{2}-T_{1}\right)^{-1} \int_{T_{1}}^{T_{2}} P_{T} d T
$$

at the maturity $T_{1}$, whose time- $t$ price $F_{t}\left(T_{1}, T_{2}\right)$ is to be paid at $T_{1}$.

The following observation is crucial: According to formula 2.2, the payoff (3.4) can be expressed in terms of prices of fuels, so that in our model the forward contract on electricity can be viewed as a forward contract on fuels and since the classical no-arbitrage theory makes sense on the market of fuels, it can also be used 
to price electricity derivatives such as (3.4). In other terms, our production-based structural model relating electricity and fuels allows us to transfer the whole no-arbitrage classical approach from fuels to electricity market, so overcoming the non-storability issue. that:

By Assumption 2.2 and classical result on forward pricing (see [8] Chapter 26), it immediately follows

$$
F_{t}\left(T_{1}, T_{2}\right)=\frac{1}{T_{2}-T_{1}} \int_{T_{1}}^{T_{2}} \frac{\mathbb{E}_{t}^{\mathbb{Q}}\left[e^{-\int_{t}^{T} r_{u} d u} P_{T}\right]}{\mathbb{E}_{t}^{\mathbb{Q}}\left[e^{-\int_{t}^{T} r_{u} d u}\right]} d T
$$

$\mathbb{E}_{t}^{\mathbb{Q}}$ denoting the conditional $\mathbb{Q}$-expectation given market's filtration $\mathcal{F}_{t}$, for $t \geq 0$.

Let $T \in\left[T_{1}, T_{2}\right]$. It is convenient for the next calculations to introduce the forward measure $\mathbb{Q}_{T}$ defined by the density

$$
\frac{d \mathbb{Q}_{T}}{d \mathbb{Q}}:=\frac{e^{-\int_{t}^{T} r_{u} d u}}{B_{t}(T)} \text { on } \mathcal{F}_{T}^{W}
$$

where

$$
B_{t}(T):=\mathbb{E}_{t}^{\mathbb{Q}}\left[e^{-\int_{t}^{T} r_{u} d u}\right]
$$

is the time- $t$ price of a zero-coupon bond with maturity $T$. Then:

$$
\begin{aligned}
F_{t}\left(T_{1}, T_{2}\right) & =\frac{1}{T_{2}-T_{1}} \int_{T_{1}}^{T_{2}} \mathbb{E}^{\mathbb{Q}_{T}}\left[P_{T} \mid \mathcal{F}_{t}\right] d T \\
& =\sum_{i=1}^{n} \frac{1}{T_{2}-T_{1}} \int_{T_{1}}^{T_{2}} \mathbb{E}^{\mathbb{Q}_{T}}\left[S_{T}^{(i)} \mathbf{1}_{\left\{D_{T} \in I_{i}^{\pi_{T}}(T)\right\}} \mid \mathcal{F}_{t}\right] d T
\end{aligned}
$$

We denote by $\Pi_{n}$ the set of all permutations over the index set $\{1, \ldots, n\}$. Let $\pi \in \Pi_{n}$ be a given non-random permutation. Under the assumption $S_{t}^{i} \in L^{1}\left(\mathbb{Q}_{t}\right)$ for any $t \geq 0$ and $1 \leq i \leq n$, we can define the following changes of probability on $\mathcal{F}_{T}^{W}$ :

$$
\frac{d \mathbb{Q}_{T}^{i}}{d \mathbb{Q}_{T}}=\frac{S_{T}^{i}}{\mathbb{E}^{\mathbb{Q}_{T}}\left[S_{T}^{i}\right]}, \quad 1 \leq i \leq n, T \leq T^{*}
$$

Proposition 3.1 If our model assumptions hold and if $S_{T}^{i} \in L^{1}\left(\mathbb{Q}_{T}\right)$ for all $T \in\left[T_{1}, T_{2}\right]$ and $1 \leq i \leq n$, we have

$$
F_{t}\left(T_{1}, T_{2}\right)=\frac{1}{T_{2}-T_{1}} \sum_{i=1}^{n} \sum_{\pi \in \Pi_{n}} \int_{T_{1}}^{T_{2}} F_{t}^{\pi(i)}(T) \mathbb{Q}_{T}^{\pi(i)}\left[\pi_{T}=\pi \mid \mathcal{F}_{t}^{W}\right] \mathbb{Q}_{T}\left[D_{T} \in I_{i}^{\pi}(T) \mid \mathcal{F}_{t}^{0, \Delta}\right] d T
$$

for $t \in\left[0, T_{1}\right]$, where $F_{t}^{i}(T)$ denotes the price at time $t$ of forward contract on the $i$-th commodity with maturity $T$ and $\mathcal{F}_{t}^{0, \Delta}$ is the natural filtration generated by both $W^{0}$ and $\Delta$.

Proof. Notice first that

$$
F_{t}\left(T_{1}, T_{2}\right)=\frac{1}{T_{2}-T_{1}} \int_{T_{1}}^{T_{2}} F_{t}(T) d T
$$

where $F_{t}(T)=\mathbb{E}^{\mathbb{Q}_{T}}\left[P_{T} \mid \mathcal{F}_{t}\right]$ can be interpreted as the $t$-price of a forward contract with maturity $T$ and instantaneous delivery at maturity. By the definition of electricity forward price $F_{t}(T)$, we have

$$
\begin{aligned}
F_{t}(T) & =\sum_{i=1}^{n} \mathbb{E}^{\mathbb{Q}_{T}}\left[S_{T}^{(i)} \mathbf{1}_{\left\{D_{T} \in I_{i}^{\pi_{T}}(T)\right\}} \mid \mathcal{F}_{t}\right] \\
& =\sum_{i=1}^{n} \sum_{\pi \in \Pi_{n}} \mathbb{E}^{\mathbb{Q}_{T}}\left[S_{T}^{\pi(i)} \mathbf{1}_{\left\{D_{T} \in I_{i}^{\pi}(T)\right\}} \mathbf{1}_{\left\{\pi_{T}=\pi\right\}} \mid \mathcal{F}_{t}\right] .
\end{aligned}
$$


If we use the mutual (conditional) independence between $W, W^{0}$ and $\Delta$ as in Remark 2, we get

$$
F_{t}(T)=\sum_{i=1}^{n} \sum_{\pi \in \Pi_{n}} \mathbb{E}^{\mathbb{Q}_{T}}\left[S_{T}^{\pi(i)} \mathbf{1}_{\left\{\pi_{T}=\pi\right\}} \mid \mathcal{F}_{t}^{W}\right] \mathbb{Q}_{T}\left[D_{T} \in I_{i}^{\pi}(T) \mid \mathcal{F}_{t}^{0, \Delta}\right]
$$

Using the change of probability $d \mathbb{Q}_{T}^{\pi(i)} / d \mathbb{Q}_{T}$ yields

$$
\mathbb{E}^{\mathbb{Q}_{T}}\left[S_{T}^{\pi(i)} \mathbf{1}_{\left\{\pi_{T}=\pi\right\}} \mid \mathcal{F}_{t}^{W}\right]=F_{t}^{\pi(i)}(T) \mathbb{Q}_{T}^{\pi(i)}\left[\pi_{T}=\pi \mid \mathcal{F}_{t}^{W}\right],
$$

so giving, after integrating between $T_{1}$ and $T_{2}$ and dividing by $T_{2}-T_{1}$, the announced formula.

The main formula (3.8) provides a formal expression to the current intuition of electricity market players that the forward prices are expected to be equal to a weighted average of forward prices of fuels. Such weights are determined by the crossing of the expected demand with the expected stack curve of the technologies. We will see in Section 5 that this model is able to explain the spikes of electricity. Nonetheless, we can already observe that formula (3.8) reproduces the stylized fact that the paths of electricity forward prices are much smoother than those of spot prices. This is due to the averaging effect of the conditional expectation on the indicator functions appearing in formula (2.2), even in the degenerate case when the delivery period reduces to a singleton.

In the next section, we will perform some explicit computations of the conditional probabilities involved in the previous formula for electricity forward prices, under more specific assumptions on the dynamics of prices and demand.

\section{A model with two technologies and constant coefficients}

In order to push further the explicit calculations, we assume now that the volatilities of fuels are constant, i.e. $\sigma_{t}^{i, j}=\sigma^{i, j}$ for some constant numbers $\sigma^{i, j}>0,1 \leq i, j \leq n$, and that the interest rate is constant $r_{t}=r>0$. Under the latter simplification, the forward-neutral measures $\mathbb{Q}_{T}$ all coincide with the minimal martingale measure $\mathbb{Q}=\mathbb{Q}^{\text {min }}$. Similar closed-form expressions can be obtained by assuming a Gaussian Heath-Jarrow-Morton model for the yield curve.

Let us assume from now on that only two technologies are available, i.e. $n=2$.

Dynamics of capacity processes $\Delta^{i}$. In order to get explicit formulae for forward prices we have to specify the dynamics of each capacity process $\Delta^{i}$. We assume that the probability space $(\Omega, \mathcal{F}, \mathbb{P})$ supports four (independent) standard Poisson processes $N_{t}^{1, u}, N_{t}^{1, d}, N_{t}^{2, u}$ and $N_{t}^{2, d}$ with constant intensities $\lambda_{1}^{u}, \lambda_{1}^{d}, \lambda_{2}^{u}, \lambda_{2}^{d}>0$ and we assume that each $\Delta^{i}$ follows

$$
d \Delta_{t}^{i}=\left(m_{i}-M_{i}\right) \mathbf{1}_{\left(\Delta_{t}^{i}=M_{i}\right)} d N_{t}^{i, d}+\left(M_{i}-m_{i}\right) \mathbf{1}_{\left(\Delta_{t}^{i}=m_{i}\right)} d N_{t}^{i, u}, \quad \Delta_{0}^{i}=M_{i}
$$

Remark 4 Basically we are assuming that each capacity $i$ can take only two values $M_{i}>m_{i}$ and it switches from $m_{i}$ to $M_{i}$ (resp. from $M_{i}$ to $m_{i}$ ) when the process $N^{i, u}$ (resp. $N^{i, d}$ ) jumps. Each capacity evolves independently of each other. At $t=0$ both technologies have maximal capacity $M_{i}$. The fact that the intensities of upside and downside jumps of $\Delta^{i}$ are not necessarily equal introduces a skewness in the probability of being at capacity $M_{i}$ or $m_{i}$.

Let $T$ be any time in the delivery period $\left[T_{1}, T_{2}\right]$. First observe that, since $\Delta$ is independent of $W^{0}$ and its law is invariant under the probability change from $\mathbb{P}$ to $\mathbb{Q}=\mathbb{Q}_{T}$ as in Remark 2 , we have $\mathbb{Q}_{T}\left[\Delta_{T}^{\pi(1)}=\right.$ $\left.x_{1} \mid \mathcal{F}_{t}^{0, \Delta}\right]=\mathbb{P}\left[\Delta_{T}^{\pi(1)}=x_{1} \mid \Delta_{t}\right]$ as well as

$$
\mathbb{Q}_{T}\left[\Delta_{T}^{\pi(1)}=x_{1}, \Delta_{T}^{\pi(2)}=x_{2} \mid \mathcal{F}_{t}^{0, \Delta}\right]=\mathbb{P}\left[\Delta_{T}^{\pi(1)}=x_{1}, \Delta_{T}^{\pi(2)}=x_{2} \mid \Delta_{t}\right]
$$


for $x_{1} \in\left\{m_{1}, M_{1}\right\}$ and $x_{2} \in\left\{m_{2}, M_{2}\right\}$.

As a consequence of the previous assumption on the dynamics of capacities $\Delta^{i}$, the conditional probabilities $\mathbb{Q}_{T}\left[D_{T} \in I_{k}^{\pi}(T) \mid \mathcal{F}_{t}^{0, \Delta}\right]$ appearing in the main formula (3.8) can be decomposed as follows

$$
\begin{aligned}
\mathbb{Q}_{T}\left[D_{T} \in I_{1}^{\pi}(T) \mid \mathcal{F}_{t}^{0, \Delta}\right]= & \mathbb{Q}_{T}\left[D_{T} \leq \Delta_{T}^{\pi(1)} \mid \mathcal{F}_{t}^{0, \Delta}\right] \\
= & \mathbb{P}\left[\Delta_{T}^{\pi(1)}=m_{1} \mid \mathcal{F}_{t}^{\Delta}\right] \mathbb{Q}_{T}\left[D_{T} \leq m_{1} \mid \mathcal{F}_{t}^{0}\right] \\
& +\mathbb{P}\left[\Delta_{T}^{\pi(1)}=M_{1} \mid \mathcal{F}_{t}^{\Delta}\right] \mathbb{Q}_{T}\left[D_{T} \leq M_{1} \mid \mathcal{F}_{t}^{0}\right]
\end{aligned}
$$

A similar decomposition for $\mathbb{Q}_{T}\left[D_{T} \in I_{2}^{\pi}(T) \mid \mathcal{F}_{t}^{0, \Delta}\right]$ holds too. It is clear now that the building blocks appearing in such formulae are the probabilities $\mathbb{P}\left[\Delta_{T}^{k}=x \mid \Delta_{t}^{k}\right]$ and $\mathbb{Q}_{T}\left[D_{T} \leq y \mid \mathcal{F}_{t}^{0}\right]$.

It remains to compute $\mathbb{P}\left[\Delta_{T}^{k}=x \mid \mathcal{F}_{t}^{\Delta}\right]$ for $k=1,2$ and $x=M_{k}, m_{k}$. As an example, we will compute $\mathbb{P}\left[\Delta_{T}^{k}=m_{k} \mid \Delta_{0}=M_{k}\right]$. For the sake of simplicity, we will drop for a while the index $k$ from the notation, that is we will write $\Delta_{T}$ for $\Delta_{T}^{k}, M$ for $M_{k}$, and so on.

Let $\tau^{d}$ be the last jump time of the process $N_{t}^{d}$ before $T$, i.e. $\tau^{d}=\sup \left\{t \in[0, T]: \Delta N_{t}^{d}=1\right\}$ with the convention that $\sup \emptyset=0$. Notice that on the event $\left\{\tau^{d}>0\right\}$ we have $\left\{\Delta_{T}=m\right\}=\left\{N_{\tau^{d}}^{u}=N_{T}^{u}\right\}$. On the other hand, on the set $\left\{\tau^{d}=0\right\}$ the process $\Delta$ has no jump downwards over the time interval $[0, T]$, so that $\mathbb{P}\left(\Delta_{T}=m, \tau^{d}=0 \mid \Delta_{0}=M\right)=0$. Using the independence between $N^{d}$ and $N^{u}$ and the stationarity of $N^{u}$, one has

$$
\begin{aligned}
\mathbb{P}\left[\Delta_{T}=m \mid \Delta_{0}=M\right] & =\mathbb{E}\left[\mathbb{P}\left(N_{\tau^{d}}^{u}=N_{T}^{u} \mid \tau^{d}\right) \mathbf{1}_{\tau^{d}>0}\right] \\
& =\mathbb{E}\left[\mathbb{P}\left(N_{T-\tau^{d}}^{u}=0 \mid T-\tau^{d}\right) \mathbf{1}_{T-\tau^{d}<T}\right] \\
& =\mathbb{E}\left[e^{-\lambda^{u}\left(T-\tau^{d}\right)} \mathbf{1}_{T-\tau^{d}<T}\right] .
\end{aligned}
$$

By the time-reversal property of the standard Poisson process $^{1}$, the random variable $T-\tau^{d}$ has the same law as $T_{1}^{d} \wedge T$, where $T_{1}^{d}$ is the first jump time of $\left(N_{t}^{d}\right)_{t \geq 0}$. We recall that $T_{1}$ has exponential law with parameter $\lambda^{d}$. Thus we have

$$
\begin{aligned}
\mathbb{P}\left[\Delta_{T}=m \mid \Delta_{0}=M\right] & =\mathbb{E}\left[e^{-\lambda^{u}\left(T_{1}^{d} \wedge T\right)} \mathbf{1}_{T_{1}^{d}<T}\right]=\mathbb{E}\left[e^{-\lambda^{u} T_{1}^{d}} \mathbf{1}_{T_{1}^{d}<T}\right] \\
& =\frac{\lambda^{d}}{\lambda^{d}+\lambda^{u}}\left(1-e^{-\left(\lambda^{d}+\lambda^{u}\right) T}\right)
\end{aligned}
$$

The general result follows by stationarity :

$$
\mathbb{P}\left[\Delta_{T}^{k}=m_{k} \mid \Delta_{t}^{k}=M_{k}\right]=\frac{\lambda_{k}^{d}}{\lambda_{k}^{d}+\lambda_{k}^{u}}\left(1-e^{-\left(\lambda_{k}^{d}+\lambda_{k}^{u}\right)(T-t)}\right), \quad k=1,2 .
$$

Using the same arguments, one can obtain similar expressions for the remaining probabilities $\mathbb{P}\left[\Delta_{T}^{k}=\right.$ $\left.x \mid \mathcal{F}_{t}^{\Delta}\right]$ for $k=1,2$ and $x=M_{k}, m_{k}$.

Dynamics of the electricity demand $D$. We also assume that the residual demand is defined by the a mean-reverting Ornstein-Uhlenbeck process. It is well-known that this process has a positive probability to be negative. Nonetheless, in the empirical study, it will applied to a residual demand, which can be negative (see Section 2).

$$
d D_{t}=a\left(b(t)-D_{t}\right) d t+\delta d W_{t}^{0} \quad, D_{0}>0
$$

for given strictly positive constants $a$ and $\delta$, and a long-term mean $b(t)$ which can vary with time, to incorporate annual seasonal effects as in [2] :

$$
b(t)=b_{0}+b_{1} \cos \left(2 \pi t-b_{2}\right)-\frac{2 \pi}{a} \sin \left(2 \pi t-b_{2}\right),
$$

\footnotetext{
${ }^{1}$ The process $\left(N_{T}^{d}-N_{(T-t)-}^{d}\right)_{t \geq 0}$ as the same law as $\left(N_{t}^{d}\right)_{t \geq 0}$.
} 
where $b_{0}, b_{1}$ and $b_{2}$ are (positive) constants. Then we set $\tilde{b}(t)=b_{0}+b_{1} \cos \left(2 \pi t-b_{2}\right)$. In this case, there are explicit formulae for $\mathbb{Q}\left[D_{T} \leq x_{1} \mid \mathcal{F}_{t}^{0}\right]$ and $\mathbb{Q}\left[x_{1}<D_{T} \leq x_{1}+x_{2} \mid \mathcal{F}_{t}^{0}\right]$, for any $0 \leq t \leq T$ and $x_{1}, x_{2} \in \mathbb{R}$, given by

$$
\begin{aligned}
\mathbb{Q}\left[D_{T} \leq x_{1} \mid \mathcal{F}_{t}^{0}\right]= & \Phi\left(\frac{x_{1}-\tilde{b}(T)-\left(D_{t}-\tilde{b}(t)\right) e^{-a(T-t)}}{\delta \sqrt{\frac{1}{2 a}\left(1-e^{-2 a(T-t)}\right)}}\right) \\
\mathbb{Q}\left[x_{1}<D_{T} \leq x_{1}+x_{2} \mid \mathcal{F}_{t}^{0}\right]= & \Phi\left(\frac{\left(x_{1}+x_{2}\right)-\tilde{b}(T)-\left(D_{t}-\tilde{b}(t)\right) e^{-a(T-t)}}{\delta \sqrt{\frac{1}{2 a}\left(1-e^{-2 a(T-t)}\right)}}\right) \\
& -\Phi\left(\frac{x_{1}-\tilde{b}(T)-\left(D_{t}-\tilde{b}(t)\right) e^{-a(T-t)}}{\delta \sqrt{\frac{1}{2 a}\left(1-e^{-2 a(T-t)}\right)}}\right),
\end{aligned}
$$

where $\Phi$ denotes the cumulative distribution function of an $\mathcal{N}(0,1)$ random variable.

Let $T \in\left[T_{1}, T_{2}\right]$. The next step consists in computing the law of the couple $\left(S_{T}^{1}, S_{T}^{2}\right)$ under each probability $\mathbb{Q}_{T}^{\pi(i)}$ for any permutation $\pi \in \Pi_{2}$ and any $i=1,2$, in order to get an explicit expression for the conditional probability $\mathbb{Q}_{T}\left[\pi_{T}=\pi \mid \mathcal{F}_{t}^{W}\right]=\mathbb{Q}\left[\pi_{T}=\pi \mid \mathcal{F}_{t}^{W}\right]$ appearing in formula (3.8). It can be easily done in this setting by using multidimensional Girsanov's theorem (see, e.g., Karatzas and Shreve's book [21], Theorem 5.1 in Chapter 3). Indeed, if we denote $\sigma^{i}$ the 2-dimensional vector $\left(\sigma^{i, 1}, \sigma^{i, 2}\right)$ and we set

$$
Z_{t}^{i}:=\left.\frac{d \mathbb{Q}_{T}^{i}}{d \mathbb{Q}}\right|_{\mathcal{F}_{t}^{W}}
$$

we get that

$$
Z_{t}^{i}=\exp \left\{\sigma^{i} \cdot W_{t}^{\mathbb{Q}}-\frac{1}{2}\left\|\sigma^{i}\right\|^{2} t\right\}, \quad t \in[0, T] .
$$

A simple application of Girsanov's theorem provides the following $\mathbb{Q}_{T}^{i}$-dynamics of each price process $S^{j}$ for $j=1,2$ :

$$
S_{t}^{j}=S_{0}^{j} \exp \left\{\left(r-\frac{1}{2}\left\|\sigma^{j}\right\|^{2}+\sigma^{j} \cdot \sigma^{i}\right) t+\sigma^{j} \cdot \widehat{W}_{t}\right\}, \quad t \in[0, T],
$$

where $\widehat{W}=\left(\widehat{W}^{1}, \widehat{W}^{2}\right)$ is a 2-dimensional Brownian motion under $\mathbb{Q}_{T}^{i}$. The following result follows from direct calculation.

Proposition 4.1 Let $T_{2}>T_{1}>0$. Under our model assumptions, the price at time $t$ of an electricity forward contract with maturity $T_{1}$ and delivery period $\left[T_{1}, T_{2}\right]$, denoted by $F_{t}\left(T_{1}, T_{2}\right)$, is given by the following formula:

$$
F_{t}\left(T_{1}, T_{2}\right)=\sum_{\pi \in \Pi_{2}} \frac{1}{T_{2}-T_{1}} \int_{T_{1}}^{T_{2}}\left(A_{1}(t, T)+A_{2}(t, T)\right) d T,
$$

where

$$
\begin{aligned}
A_{1}(t, T):= & \sum_{\left\{x_{1}=m_{\pi(1)}, M_{\pi(1)}\right\}} F_{t}^{\pi(1)}(T) \mathbb{Q}_{T}^{\pi(1)}\left[\pi_{T}=\pi \mid \mathcal{F}_{t}^{W}\right] \mathbb{P}\left[\Delta_{T}^{\pi(1)}=x_{1} \mid \Delta_{t}\right] \mathbb{Q}\left[D_{T} \leq x_{1} \mid \mathcal{F}_{t}^{0}\right] \\
A_{2}(t, T):= & \sum_{\substack{\left\{x_{1}=m_{\pi(1)}, M_{\pi(1)} ; \\
x_{2}=m_{\pi(2)}, M_{\pi(2)}\right\}\\
}} F_{t}^{\pi(2)}(T) \mathbb{Q}_{T}^{\pi(2)}\left[\pi_{T}=\pi \mid \mathcal{F}_{t}^{W}\right] \mathbb{P}\left[\Delta_{T}^{\pi(1)}=x_{1}, \Delta_{T}^{\pi(2)}=x_{2} \mid \Delta_{t}\right] \\
& \times \mathbb{Q}\left[x_{T} \leq x_{1}+x_{2} \mid \mathcal{F}_{t}^{0}\right]
\end{aligned}
$$


where, for any $\pi \in \Pi_{2}$ and $i=1,2$, the conditional probabilities $\mathbb{Q}\left[D_{T} \leq x_{1} \mid \mathcal{F}_{t}^{0}\right]$ and $\mathbb{Q}\left[x_{1}<D_{T} \leq x_{1}+x_{2} \mid \mathcal{F}_{t}^{0}\right]$ are given by (4.12) and (4.13), and

$$
\mathbb{Q}_{T}^{\pi(i)}\left[\pi_{T}=\pi \mid \mathcal{F}_{t}^{W}\right]=1-\Phi(m(t) / \gamma(t)),
$$

where $m(t)$ and $\gamma(t)$ are defined as follows:

$$
\begin{aligned}
& m(t)=\ln \frac{S_{t}^{\pi(1)}}{S_{t}^{\pi(2)}}-\left(\frac{1}{2}\left\|\sigma^{\pi(1)}-\sigma^{\pi(2)}\right\|^{2}-\left(\sigma^{\pi(1)}-\sigma^{\pi(2)}\right) \cdot \sigma^{\pi(i)}\right)(T-t) \\
& \gamma^{2}(t)=\left\|\sigma^{\pi(1)}-\sigma^{\pi(2)}\right\|^{2}(T-t) .
\end{aligned}
$$

Proof. It suffices to combine the different formulae obtained in this section and observe that for any $\pi \in \Pi_{2}$ and $i=1,2$ we have

$$
\mathbb{Q}_{T}^{\pi(i)}\left[\pi_{T}=\pi \mid \mathcal{F}_{t}^{0}\right]=\mathbb{Q}_{T}^{\pi(i)}\left[S_{T}^{\pi(1)} \leq S_{T}^{\pi(2)} \mid \mathcal{F}_{T}^{W}\right]=\mathbb{Q}_{T}^{\pi(i)}\left[X \leq 0 \mid \mathcal{F}_{t}^{W}\right]
$$

where $X:=\ln \left(S_{T}^{\pi(1)} / S_{T}^{\pi(2)}\right)$. Under $\mathbb{Q}_{T}^{\pi(i)}$,

$$
\begin{aligned}
X= & \ln \frac{S_{t}^{\pi(1)}}{S_{t}^{\pi(2)}}+\sum_{j=1}^{2}\left(\sigma^{\pi(1), j}-\sigma^{\pi(2), j}\right)\left(\widehat{W}_{T}^{j}-\widehat{W}_{t}^{j}\right) \\
& -\sum_{j=1}^{2}\left(\frac{1}{2}\left(\left(\sigma^{\pi(1), j}\right)^{2}-\left(\sigma^{\pi(2), j}\right)^{2}\right)-\left(\sigma^{\pi(1), j}-\sigma^{\pi(2), j}\right) \sigma^{\pi(i), j}\right)(T-t) .
\end{aligned}
$$

Thus, conditioned to $\mathcal{F}_{t}^{W}$, the random variable $X$ is normal with mean $m(t)$ and variance $\gamma^{2}(t)$, where

$$
m(t)=\ln \frac{S_{t}^{\pi(1)}}{S_{t}^{\pi(2)}}-\sum_{j=1}^{2}\left(\frac{1}{2}\left(\left(\sigma^{\pi(1), j}\right)^{2}-\left(\sigma^{\pi(2), j}\right)^{2}\right)-\left(\sigma^{\pi(1), j}-\sigma^{\pi(2), j}\right) \sigma^{\pi(i), j}\right)(T-t)
$$

and

$$
\gamma^{2}(t)=\sum_{j=1}^{2}\left(\sigma^{\pi(1), j}-\sigma^{\pi(2), j}\right)^{2}(T-t) .
$$

Notice that only the mean $m(t)$ depends on $\pi(i)$. Finally, we have

$$
\begin{aligned}
\mathbb{Q}_{T}^{\pi(i)}\left[\pi_{T}=\pi \mid \mathcal{F}_{t}^{W}\right] & =\mathbb{Q}_{T}^{\pi(i)}\left[X \leq 0 \mid \mathcal{F}_{t}^{W}\right] \\
& =\mathbb{Q}_{T}^{\pi(i)}\left[(X-m(t)) / \gamma(t) \leq-m(t) / \gamma(t) \mid \mathcal{F}_{t}^{W}\right] \\
& =\Phi(-m(t) / \gamma(t))=1-\Phi(m(t) / \gamma(t)),
\end{aligned}
$$

where $\Phi$ is the c.d.f. of a standard gaussian random variable. The proof is complete.

\section{$5 \quad$ Numerical results}

To provide a coherent and tractable framework for numerical examples, we follow the two fuels model of the previous section and we push further the simplification.

Data choice. We test the model on the French deregulated power market. The data cover the period going from January, $1^{\text {st }}, 2007$ to December, $31^{\text {st }}, 2008$. For the demand process $\left(D_{t}\right)$, we used the data provided by the French TSO, RTE, on its web site. ${ }^{2}$ The hourly demand can be retrieved. The two technologies

\footnotetext{
2RTE: www.rte-france.fr
} 
we have chosen are natural gas plants and fuel combustion turbines. They are known to frequently determine the spot price during peaking hours, since they are the most expensive ones. Moreover, a decomposition of the production is provided by RTE for each type of generation asset (nuclear, hydrolic plants, coal and gas, fuels, peak). Hence, it allowed us to deduce the residual demand addressed to gas and fuels technologies by substracting the nuclear and hydrolic production from the demand. Since these two technologies are setting the price during peaking hours, we focus our analysis on one particular hour of the day. We have chosen the $12^{\text {th }}$ hour, which is usualy the first peaking hour of the day (the next one being $19^{\text {th }}$ hour). The electricity spot and future prices are provided by Powernext. The $\mathrm{CO}_{2}$ prices are provided by PointCarbon data. For fuel and gas prices, we used Platt's data. Gas prices are quoted in GBP and fuel prices en USD. We used the daily exchange rate to convert GBP into EUR.

Reconstruction of $S_{t}^{1}$ and $S_{t}^{2}$. In our model, we need to rebuild the spot prices of the two technologies $S_{t}^{1}$ and $S_{t}^{2}$. To tackle with the problem of aggregating the numerous gas and fuel power plants into only two technologies, we used the information provided by the French Ministry of Industry on electricity production costs ${ }^{3}$. It gives an average heat rate for each techology. We use also an average emission rate for $\mathrm{CO}_{2}$ emissions of each technology. Furthermore, for fuel power plants production costs, one need to take into account the transportation cost from ARA zone to the location of the plants. We use an average fixed cost. Thus, we obtain the following expressions for the prices of the two technologies :

$$
\left\{\begin{array}{l}
S_{t}^{1}=101.08 \cdot S_{t}^{g}+0.49 \cdot S_{t}^{c o_{2}} \\
S_{t}^{2}=0.38 \cdot S_{t}^{f}+0.88 \cdot S_{t}^{c o_{2}}+13.44
\end{array}\right.
$$

where $S^{g}, S^{f}$ and $S^{c o_{2}}$ denote respectively gas (€/therm), fuel and carbon emission prices (€/ton).

Remark 5 One can observe that the ordering between the two technologies never changes on historical data. Fuel combustion turbines are known to be more expensive than gas plants. If the prices of technologies follow the dynamics given by (2.1), the probability to have different orders $\pi(t) \in \Pi$ can be positive. Nevertheless, for a reasonable choice of parameters, this probability can be made sufficiently small. Hence, we make the rough approximation that $\mathbb{P}\left(S_{t}^{1}<S_{t}^{2}\right)=1$ for all $t$.

Estimation of electricity demand. The demand process given by expression (4.11) is estimated via the Maximum Likelihood Principle. Let's remind that the demand process is given by :

$$
D_{t}=\tilde{b}(t)+X_{t}=b_{0}+b_{1} \cos \left(2 \pi t-b_{2}\right)+X_{t}
$$

where $X_{t}$ is an Ornstein-Uhlenbeck process with a known Likelihood expression (see [1], Section 5). For a discrete sample $\left(D_{t_{1}}, \ldots, D_{t_{n}}\right)$ observed at fixed times with a constant time step $\left(t_{i}-t_{i-1}\right)=\Delta t, i=1 \ldots n$, an expression of the Likelihood is

$$
\mathcal{L}\left(b_{0}, b_{1}, b_{2}, a, \delta, D_{t_{1}}, \ldots, D_{t_{n}}\right)=\frac{1}{(\sqrt{2 \pi} v)^{n}} \exp \left(-\frac{1}{2 v} \sum_{i=1}^{n-1}\left(\left(D_{t_{i+1}}-\tilde{b}\left(t_{i+1}\right)\right)-e^{a \Delta t}\left(D_{t_{i}}-\tilde{b}\left(t_{i}\right)\right)\right)^{2}\right),
$$

where $v=\delta^{2} \frac{e^{2 a \Delta t}-1}{2 a}$ and $\tilde{b}(t)$ is the same as above. We maximize numerically this expression to obtain an estimation for the set of parameters. We then test the hypothesis that each parameter is null and finally obtain the set given in Table 1 . The parameter $\hat{b}_{2}$ is not significantly different from 0 with threshold $99 \%$, thus it is taken to be zero.

Estimation of capacity process. For two technologies, the implementation of formula (2.2) is very simple. We define the following variables:

$$
R^{1}=\min \left(D_{t}^{+}, \Delta_{t}^{1}\right), \quad R^{2}=\min \left(\left(D_{t}-\Delta_{t}^{1}\right)^{+}, \Delta_{t}^{2}\right)
$$

\footnotetext{
${ }^{3}$ Ministère de l'Industrie et des Finances, www.energie.minefi.gouv.fr/energie/electric/f1e_elec.htm, see "Les coûts de référence de la production électrique"
} 


\begin{tabular}{ccccc}
$\hat{b}_{0}$ & $\hat{b}_{1}$ & $\hat{b}_{2}$ & $\hat{a}$ & $\hat{\delta}$ \\
\hline 4814 & 905 & 0 & 87.55 & 17256 \\
\hline
\end{tabular}

Table 1: Parameters estimation for the demand process.
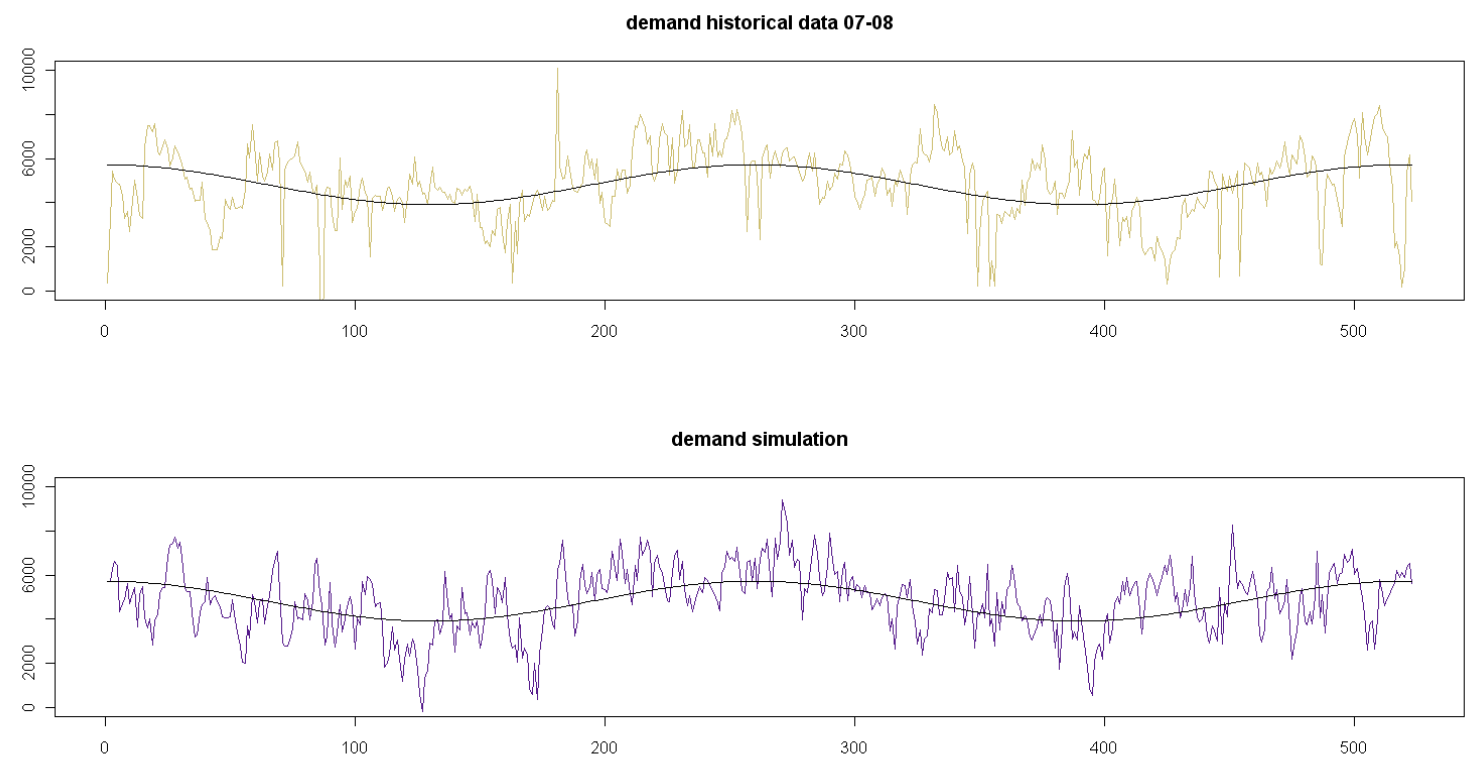

Figure 1: Midday daily demand (day-ahead peakload demand from 01/01/2007 to 31/12/2008, RTE) and simulation with fitted parameters. In black line, we showed the long trend $\tilde{b}(t)$.

where here $D_{t}$ is the sum of residual demands for the two technologies. The electricity spot price is defined by the following rule: If $R^{2}$ is positive, then we take $P=S^{2}$, and if it is zero, $P=S^{1}$. However, in our electricity spot market model, applying this rule to estimate the capacity processes $\Delta^{1}$ and $\Delta^{2}$ would lead to claiming that only the second technology (the most expensive one) is being used. Hence, to take into account all the complexity of the short-term bidding process involving production constraints (start-up cost, ramp constraints, minimal runtime...), we introduce a threshold $\bar{\Delta}^{1}$ such that the price is given by the second technology althought $R^{1}=\bar{\Delta}^{1}<\Delta^{1}$.

Noting that the inequality on $R^{1}$ is equivalent to $R^{2}>\left(\Delta^{1}-\bar{\Delta}^{1}\right)$, the threshold $\bar{\Delta}^{1}$ is obtained by solving the following program:

$$
\min _{\left(\Delta^{1}-\bar{\Delta}^{1}\right)} \sum_{i=1}^{n} \mathcal{R}\left(P_{t_{i}}-S_{t_{i}}^{1} \mathbf{1}_{\left\{R_{t_{i}}^{2} \leq\left(\Delta^{1}-\bar{\Delta}^{1}\right)\right\}}-S_{t_{i}}^{2} \mathbf{1}_{\left\{R_{t_{i}}^{2}>\left(\Delta^{1}-\bar{\Delta}^{1}\right)\right\}}\right) .
$$

The function $\mathcal{R}$ is a risk criterion: we tested two cases, the $L_{1}$ and the $L_{2}$ norms. The absolute error $\left(L_{1}\right)$ showed a global minimum and the quadratic error $\left(L_{2}\right)$ showed a local minimum on a reasonable interval (very high price peaks disturb the convergence). Thus, we use the $L_{1}$ criterion to determine that the intermediate parameter $\Delta^{1}-\bar{\Delta}^{1}$ equals $610 \mathrm{MW}$. Eventually, we have new values for $\left(D_{t}-\Delta_{t}^{1}\right) \mathbf{1}_{\left\{D_{t}>\Delta_{t}^{1}\right\}}$ and since we know exactly when $P_{t}=S_{t}^{i}$, for $i=1,2$, the estimation of the model on historical data is straightforward (see Figure 2).

Finally, we can estimate parameters for the capacity process $\Delta_{t}^{1}$ as $D_{t}=R_{t}^{1}+R_{t}^{2}$ is available. Theoretically, capacity thresholds $m_{i}$ and $M_{i}$ are structural and are known to producers. But, since they vary 


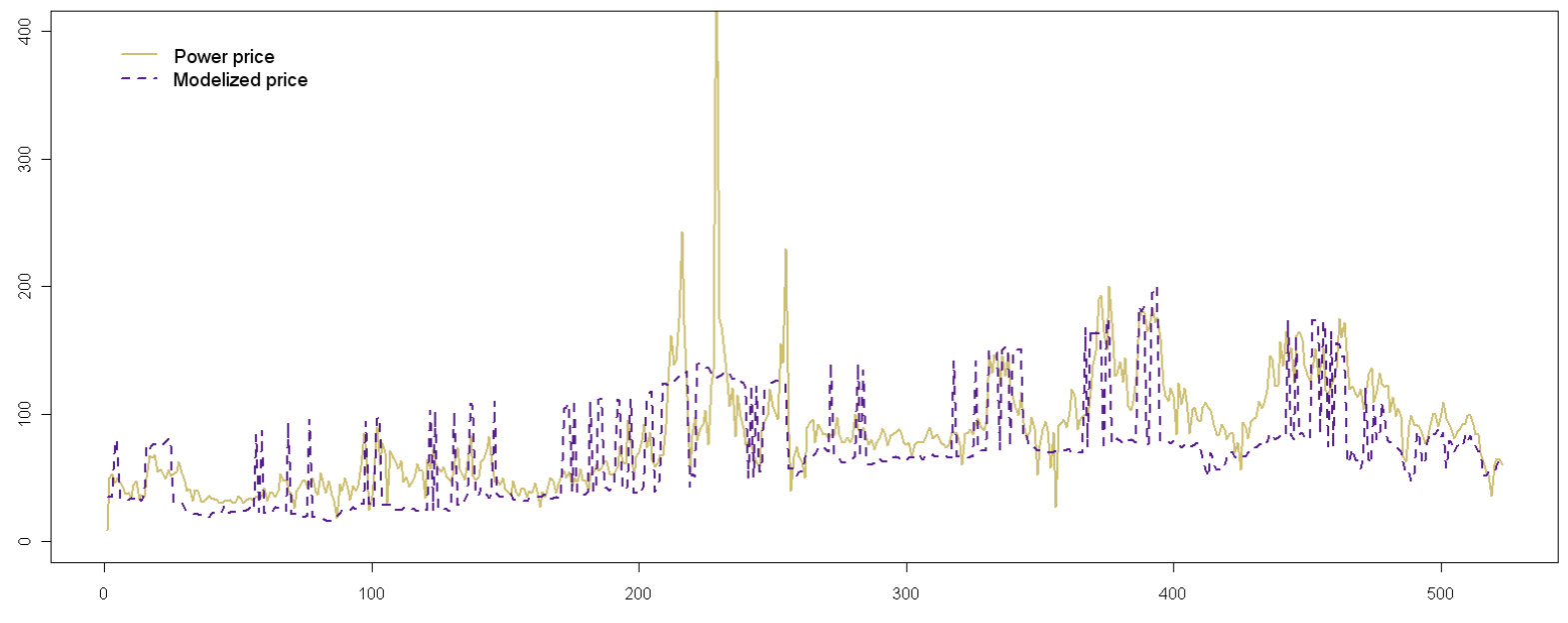

Figure 2: Midday daily prices and model fitted on historical data (POWERNEXT®) day-ahead peakload prices from $01 / 01 / 2007$ to $31 / 12 / 2008)$.

over time due to maintenance scheduling and weather conditions, we estimate their constant counterparts. Moreover, we had to deal with the fact that in our model $\Delta^{1}$ does take two values. Thus, we proceed in two steps. First, we filter the data to define a $\Delta_{t}^{1}$ taking only two values. Second, we estimate the free parameters $\lambda_{1}^{u}$ and $\lambda_{1}^{d}$ using that filtered time series.

The capacity process $\Delta^{1}$ is partially hidden, since it is observed only if $D_{t}>\Delta_{t}^{1}$. Thus, we suppose that we observe data at discrete times $t_{i}$, and we calibrate the capacity levels by minimizing the quadratic error between the series $\left(\Delta_{t_{i}}^{1} \mathbf{1}_{\left\{D_{t_{i}}>\Delta_{t_{i}}^{1}\right\}}\right)_{i=1 \ldots n}$ and two constant values, under the following structural constraints:

$$
M_{1} \geq \sup _{t \in[0, T], D_{t} \leq \Delta_{t}^{1}} D_{t} \quad ; \quad m_{1} \geq \inf _{t \in[0, T], D_{t}>\Delta_{t}^{1}} D_{t}
$$

Solving this calibration problem, we deduce the transformed serie $\tilde{\Delta}^{1}$ which takes two values:

$$
\tilde{\Delta}_{t_{i}}=m_{1} \mathbf{1}_{\left|\Delta_{t_{i}}-m_{1}\right|<\left|\Delta_{t_{i}}-M_{1}\right|}+M_{1} \mathbf{1}_{\left|\Delta_{t_{i}}-m_{1}\right| \geq\left|\Delta_{t_{i}}-M_{1}\right|}, \quad i=1 \ldots n .
$$

On that series, we estimate $\lambda_{1}^{u}$ and $\lambda_{1}^{d}$ by observing the series $\left(\tilde{\Delta}_{t_{i}}^{1} \mathbf{1}_{\left\{D_{t_{i}}>\tilde{\Delta}_{t_{i}}^{1}\right\}}\right)_{i=1 \ldots n}$. We denote $\left(t_{k(i)}\right)_{i=1 \ldots n}$ the subgrid of the discrete times where $t_{k(i)}$ is the last time before $t_{i}$ when we observe $\left(\Delta_{t_{i}}^{1}\right)_{i=1 \ldots n}$. Then, by the Bayes rule and the independence between $D_{t}$ and $\tilde{\Delta}_{t}^{1}$, the probability $\mathbb{Q}\left[\tilde{\Delta}_{t_{i}}^{1}=x \mid D_{t_{i}}>\tilde{\Delta}_{t_{i}}^{1}, \tilde{\Delta}_{t_{k(i)}}^{1}\right]$, for $i=1 \ldots n$, is given by:

$$
\mathbb{Q}_{i}[x]:=\mathbb{Q}\left[\tilde{\Delta}_{t_{i}}^{1}=x \mid D_{t_{i}}>\tilde{\Delta}_{t_{i}}, \tilde{\Delta}_{t_{k(i)}}^{1}\right]=\frac{\mathbb{P}\left[\tilde{\Delta}_{t_{i}}^{1}=x \mid \tilde{\Delta}_{t_{k(i)}}^{1}\right] \mathbb{Q}\left[D_{t_{i}}>x\right]}{\mathbb{Q}\left[D_{t_{i}}>\tilde{\Delta}_{t_{i}}^{1} \mid \tilde{\Delta}_{t_{k(i)}}^{1}\right]} .
$$

If follows that:

$$
\mathbb{Q}_{i}[x] \equiv \frac{\mathbb{P}\left[\tilde{\Delta}_{t_{i}}^{1}=x \mid \tilde{\Delta}_{t_{k(i)}}^{1}\right] \mathbb{Q}\left[D_{t_{i}}>x\right]}{\mathbb{P}\left[\tilde{\Delta}_{t_{i}}^{1}=M_{1} \mid \tilde{\Delta}_{t_{k(i)}}^{1}\right] \mathbb{Q}\left[D_{t_{i}}>M_{1}\right]+\mathbb{P}\left[\tilde{\Delta}_{t_{i}}^{1}=m_{1} \mid \tilde{\Delta}_{t_{k(i)}}^{1}\right] \mathbb{Q}\left[D_{t_{i}}>m_{1}\right]}
$$


An expression of the Likelihood for the given sample is:

$$
\mathcal{L}\left(\lambda_{1}^{u}, \lambda_{1}^{d}, \tilde{\Delta}_{t_{1}}, \ldots, \tilde{\Delta}_{t_{n}}, D_{t_{1}}, \ldots, D_{t_{n}}\right)=\prod_{i=1}^{n}\left(\mathbb{Q}_{i}[x]^{\mathbf{1}_{\left\{\tilde{\Delta}_{t_{i}}^{1}=x\right\}}}\left(1-\mathbb{Q}_{i}[x]\right)^{\left(1-\mathbf{1}_{\left\{\tilde{\Delta}_{t_{i}}^{1}=x\right\}}\right)}\right)^{\mathbf{1}_{\left\{D_{t_{i}}>\tilde{\Delta}_{t_{i}}^{1}\right\}}}
$$

We maximize this expression to obtain the intensities. The values of the parameters of the capacity process are summarized in Table 2 . We notice that $\lambda_{1}^{u}>\lambda_{1}^{d}$ means that $\mathbb{P}\left[\tilde{\Delta}_{T}^{1}=M_{1}\right]>\mathbb{P}\left[\tilde{\Delta}_{T}^{1}=m_{1}\right]$ for a sufficiently long maturity $T$.

\begin{tabular}{cccc}
$M_{1}(\mathrm{MW})$ & $m_{1}(\mathrm{MW})$ & $\lambda_{1}^{u}\left(y^{-1}\right)$ & $\lambda_{1}^{d}\left(y^{-1}\right)$ \\
\hline 5708 & 4292 & 34.78 & 24.89 \\
\hline
\end{tabular}

Table 2: Parameters for the capacity process.

A comparison with a naive econometric model. To evaluate the benefit of adding the demand and production capacity to the market model, we make a comparison between a simple econometric approach and ours. We consider the alternative linear model:

$$
P_{t}=\alpha_{0}+\alpha_{1} S_{t}^{1}+\alpha_{2} S_{t}^{2}+\epsilon_{t}
$$

where $\epsilon_{t}$ is a Gaussian white noise. We compare the linear model (5.15) to ours where we add some free linear parameters and a Gaussian noise to facilitate the comparison:

$$
P_{t}=\beta_{0}+\sum_{i=1,2} \beta_{i} S_{t}^{i} \mathbf{1}_{\left\{D_{t} \in I_{i}^{\pi_{t}}(t)\right\}}+\epsilon_{t} .
$$

In both cases, we estimate the parameters using a quadratic loss minimization. The Table 3 as well as Figure 3 shows that including explicitly demand and production capacity in the model, produces a better fit.

\begin{tabular}{lccccc} 
Price & Corr & MaxE & MAE & MSE & MPE \\
\hline Linear model & 0.756 & 406.96 & 18.35 & 919.53 & $23.734 \%$ \\
Structural Model & 0.702 & 385.23 & 17.54 & 786.20 & $23.956 \%$ \\
\hline
\end{tabular}

Table 3: Model comparison. Corr $:=$ correlation with historical price; MaxE $:=$ maximum error; MAE $:=$ mean absolute error; MSE $:=$ mean square error; $\mathrm{MPE}=$ Mean percentage error. Errors are calculated w.r.t. historical data (POWERNEXTß) day-ahead prices from 01/01/2007 to 31/12/2008).

Forward prices computation. Following the approximation given in Remark 5 , in the case of two fuels, the expression (3.8) becomes

$$
F_{t}\left(T_{1}, T_{2}\right)=\frac{1}{T_{2}-T_{1}} \int_{T_{1}}^{T_{2}} \sum_{x_{1}=m_{1}, M_{1}} \mathbb{P}\left[\Delta_{T}^{1}=x_{1} \mid \Delta_{t}\right]\left(F_{t}^{2}(T)+\left(F_{t}^{1}(T)-F_{t}^{2}(T)\right)\left(\mathbb{Q}\left[D_{T} \leq x_{1} \mid \mathcal{F}_{t}^{0}\right]\right)\right) d T .
$$

We do not have forward prices $F_{t}^{i}(T)$ at our disposal but only swap prices, i.e., values of $\frac{1}{T_{2}-T_{1}} \int_{T_{1}}^{T_{2}} F_{t}^{i}(T) d T$ for delivery periods $\left[T_{1}, T_{2}\right]$. Nevertheless, we make the approximation that:

$$
F_{t}^{i}(T) \approx \frac{1}{T_{2}-T_{1}} \int_{T_{1}}^{T_{2}} F_{t}^{i}(T) d T, \quad T \in\left[T_{1}, T_{2}\right]
$$


One may think that this approximation is quite rough for forward gas prices, since the spot market has daily granularity; but, for the prices of fuels, it is quite reasonable since spot prices take only one value per month.

We calibrate the spot price model on the former period, till June 2008, and then backtest it on future prices from July 2008 to February 2009. On that sufficiently wide interval, we focus on two assets: The two quarters ahead and three quarters ahead futures, covering Spring 2009 (April, May, June) and Summer 2009 (July, August and September). The results are illustrated on Figure 4 and Figure 5. We see that, as expected, the predicted price overestimates the real price. Indeed, we estimated the model on high peak hours of each day, which is over the mean price most of the time. However we observe strong correlation between predicted and historical prices as shown in Table 4.

\begin{tabular}{lccccccc} 
Asset & Corr & $\mathbb{E}\left[\Delta F_{t}\left(T_{1}, T_{2}\right)\right]$ & $\mathbb{V}\left[\Delta F_{t}\left(T_{1}, T_{2}\right)\right]$ & MaxE & MAE & MSE & MPE \\
\hline Spring 2009 & 0.958 & $-0.582(-0.403)$ & $2.409(1.840)$ & 49.624 & 24.815 & 851.981 & $28.297 \%$ \\
Summer 2009 & 0.939 & $-0.505(-0.402)$ & $2.174(2.014)$ & 30.928 & 11.995 & 213.484 & $12.695 \%$ \\
\hline
\end{tabular}

Table 4: Model anticipations results. Corr = correlation with historical price; $\mathbb{E}=$ yield mean (in parenthesis the real asset value); $\mathbb{V}=$ yield variance; $\mathrm{ME}=$ maximum price error; $\mathrm{MAE}=$ mean absolute error; $\mathrm{MSE}=$ mean squared error; $\mathrm{MPE}=$ mean percentage error. Errors are calculated w.r.t. historical data.)

Calibration on forward prices. The model gives two relations between the price of power and the prices of commodities. As we estimated the parameters on spot prices, we will now do the same on forward prices. Using formula (5.16), and under the previous assumptions on prices $F_{t}^{i}(T), i=1,2$, the model can be calibrated directly on forward prices. However, given the great number of parameters, we must fix some of them in order to solve the identification problem: The capacity levels $M_{1}$ and $m_{1}$, and the parameters of the demand $D_{t}$ are now fixed. Thus, the probability $\mathbb{P}\left[\Delta_{T}^{1}=x \mid \Delta_{t}\right]$ for $x=m_{1}, M_{1}$, which is integrated on the period $\left[T_{1}, T_{2}\right]$, is the only free variable. The goal is to calibrate numerically this variable on the following expression :

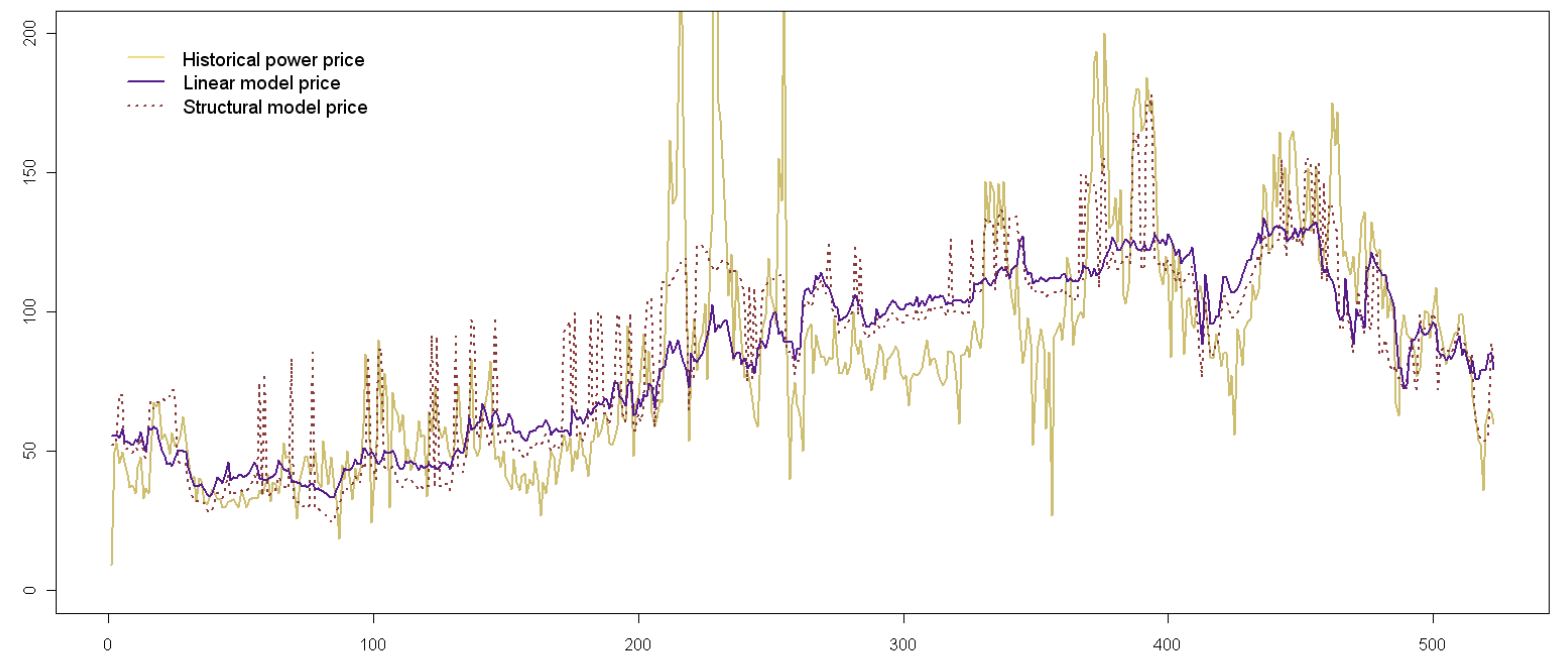

Figure 3: Prices and econometric estimation of our model and a linear model (POWERNEXT@) day-ahead prices from $01 / 01 / 2007$ to $31 / 12 / 2008$ ). 

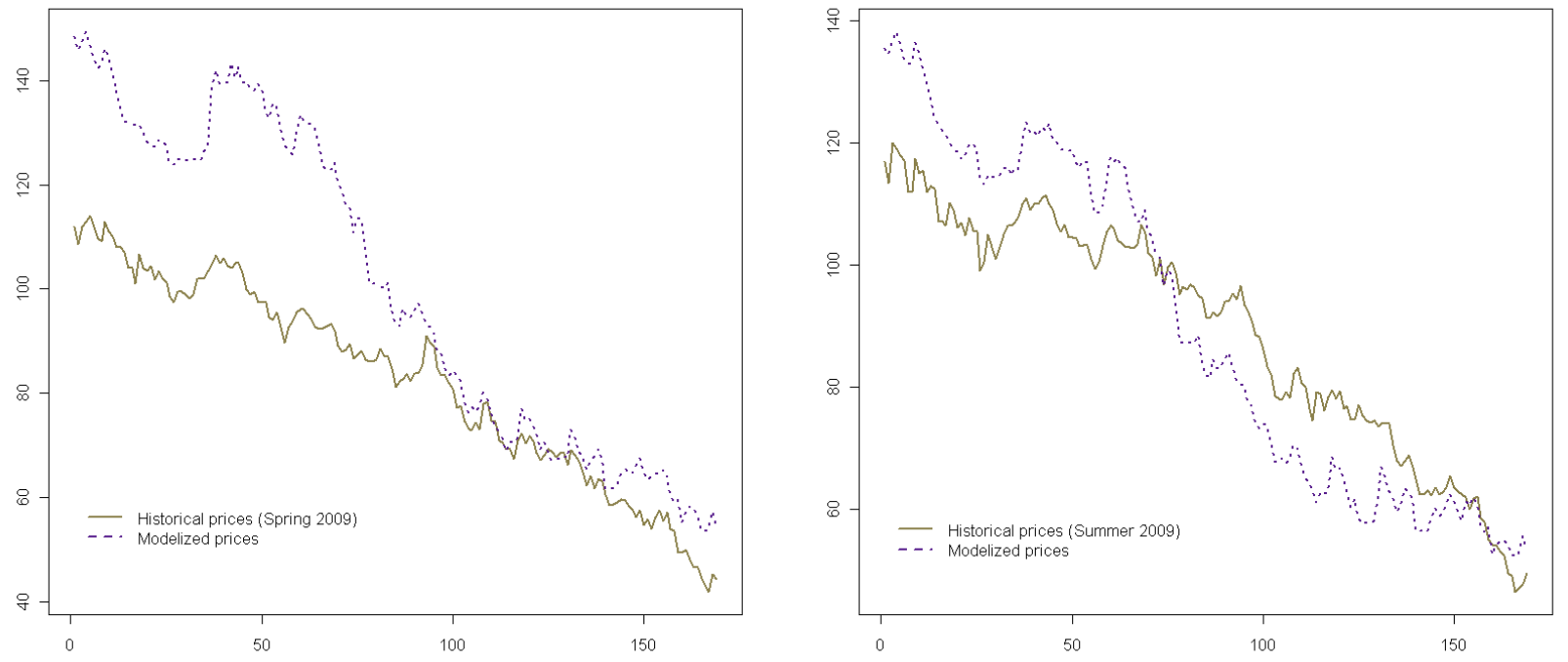

Figure 4: Forward prices : model anticipations and market data (PowERNEXT® Future prices on peak load from 01/07/2008 to 27/02/2009, 169 obs.). Left = Spring 2009; right = Summer 2009.

$$
F_{t}\left(T_{1}, T_{2}\right)=f^{1}\left(\lambda, \Delta_{t}, D_{t}\right) F_{t}^{1}\left(T_{1}, T_{2}\right)+\left(1-f^{1}\left(\lambda, \Delta_{t}, D_{t}\right)\right) F_{t}^{2}\left(T_{1}, T_{2}\right)
$$

where

$$
f^{1}\left(\lambda, \Delta_{t}, D_{t}\right)=\sum_{x=m_{1}, M_{1}} \frac{1}{T_{2}-T_{1}} \int_{T_{1}}^{T_{2}} \mathbb{P}\left[\Delta_{T}^{1}=x \mid \Delta_{t}^{1}\right] \mathbb{Q}\left[D_{T}=x \mid D_{t}\right] d T .
$$

These expressions depend on $\Delta_{t}$ and $D_{t}$ via the formulae (4.12) and (4.10). Thus, $f^{1}\left(\lambda, \Delta_{t}, D_{t}\right)$ actually depends on $t$ in an explicit manner. For the sake of simplicity, we make a few more approximations. Indeed, calibration can be difficult because of the fact that $e^{-\left(\lambda_{1}^{d}+\lambda_{1}^{u}\right)(T-t)}$ is very small when $T \gg t$. Hence, if $T \gg t$ or the parameters $\lambda$ (relation (4.10)) and $a$ (relation (4.12)) are large enough, we can make the following approximations: $\mathbb{P}\left[\Delta_{T}=x \mid \Delta_{t}\right] \cong \lim _{T \uparrow \infty} \mathbb{P}\left[\Delta_{T}=x\right]$ and $\mathbb{Q}\left[D_{T}>x \mid D_{t}\right] \cong \lim _{T \uparrow \infty} Q\left[D_{T}>x\right]$. Thus, the calibration is equivalent to a linear model estimation under constraints, whose coefficients are $f^{1}(\lambda)$ and $1-f^{1}(\lambda)$.

Under that approximation, we obtain $\mathbb{P}\left[\Delta_{T}=M_{1}\right]$ and $\mathbb{P}\left[\Delta_{T}=m\right]$, giving the expected failure probabilities for the cheapest technology on the delivery period $\left[T_{1}, T_{2}\right]$. The computation gives a sound result for calibration on Summer 2009 Future price $\left(\mathbb{P}\left[\Delta_{T}=M_{1}\right]=0.865\right)$, but not for Spring 2009 Future, which is clearly overestimated. We explain this drawback by the fact that we used the two most expensive technologies to price electricity.

Spot price simulations. Our model can be easily improved to obtain trajectories with high spikes. If the residual demand $D_{t}$ is negative, it corresponds to the case when nuclear power is the marginal unit of the system. Its cost is well-known to be constant over time (@15€/MWh). On the other hand, if the residual demand $D_{t}$ exceeds the total capacity $\Delta_{t}^{1}+\Delta_{t}^{2}$ of our two technologies, it corresponds to situations when electricity has to be imported. In the French market, which is a structural exporter, it corresponds to tension on the system and electricity is bought at high cost. This high cost is arbitrarily fixed to a constant 

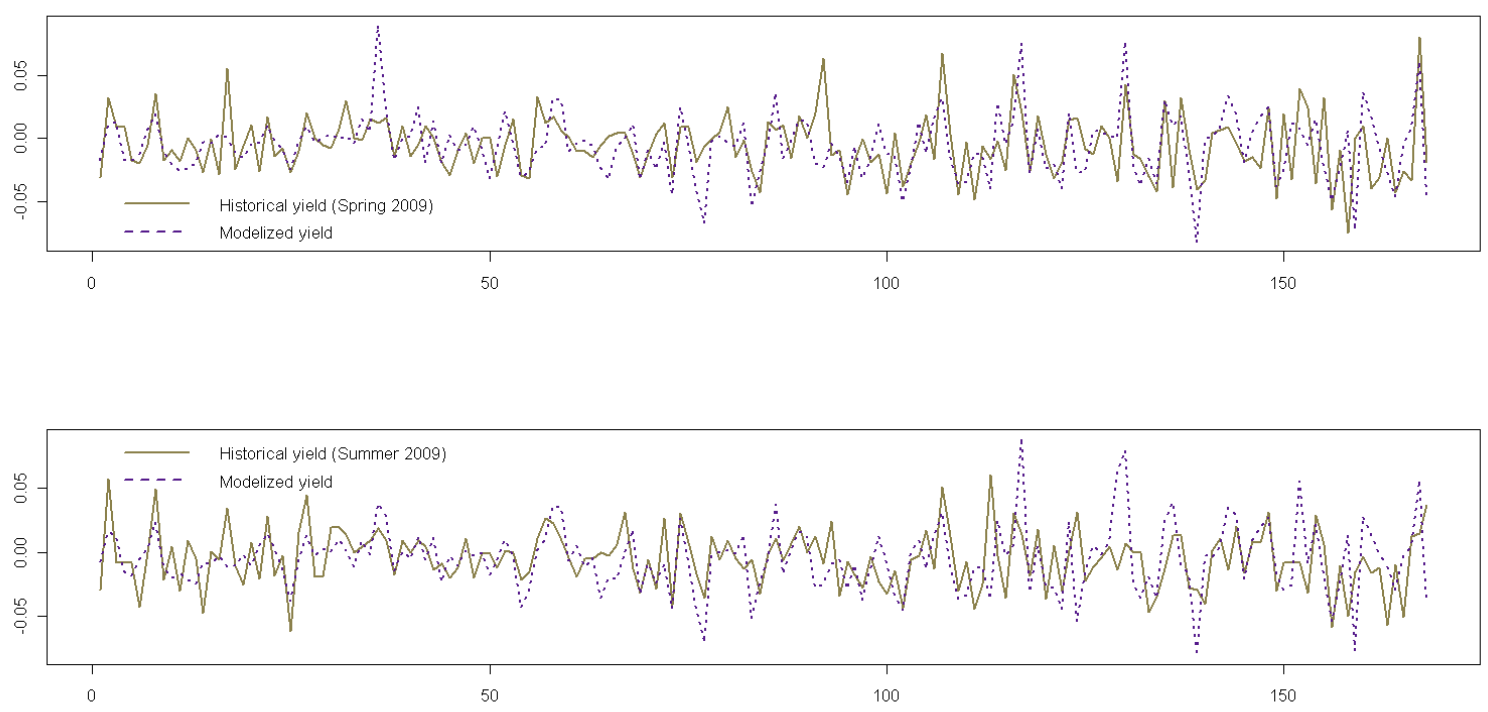

Figure 5: Forward yields : model anticipations and market data (POWERNEXT® Future yields on peak load from 01/07/2008 to 27/02/2009, 169 obs.). Up = Spring 2009; down = Summer 2009.

value (500€/MWh). In order to simulate the prices of commodities, we quickly estimate on our first sample of data (January 2007 to December 2008) the multivariate diffusion process given by the relation (2.1). The Figure 6 shows that this simple device allows us to get visible spikes.

\section{Conclusion and perspectives}

By building a market model for electricity and fuels, we provided a possible answer to the issue of pricing electricity-based derivatives using a risk-neutral approach, as in security markets. This model should be considered more as a methodology than as a definitive model for electricity spot and forward prices. Indeed, we think it may offer many perspectives for further developments. We see three different areas to explore. First, we assumed competitive equilibrium on the spot market; this assumption could be changed to take into account possible strategic bidding, so quantifying the possible deviation of forward electricity prices from their equilibrium due to frictions on the spot. Second, the spot market could be extended to a multizonal framework to take into account the fact that electricity is exchanged between different countries and that a spot price is formed in each country. Finally, the relation linking forward electricity prices to forward fuels prices could be extended to a wider class of contingent claims. We hope to develop these points in future papers.

\section{References}

[1] Y. Ait-Sahalia, Maximum likelihood estimation of discretely sampled diffusions: a closed-form approximation approach, Econometrica 70 (2002), no. 1, 223-262.

[2] M. T. Barlow, A diffusion model for electricity prices, Mathematical Finance 12 (2002), no. 4, 287-298. 


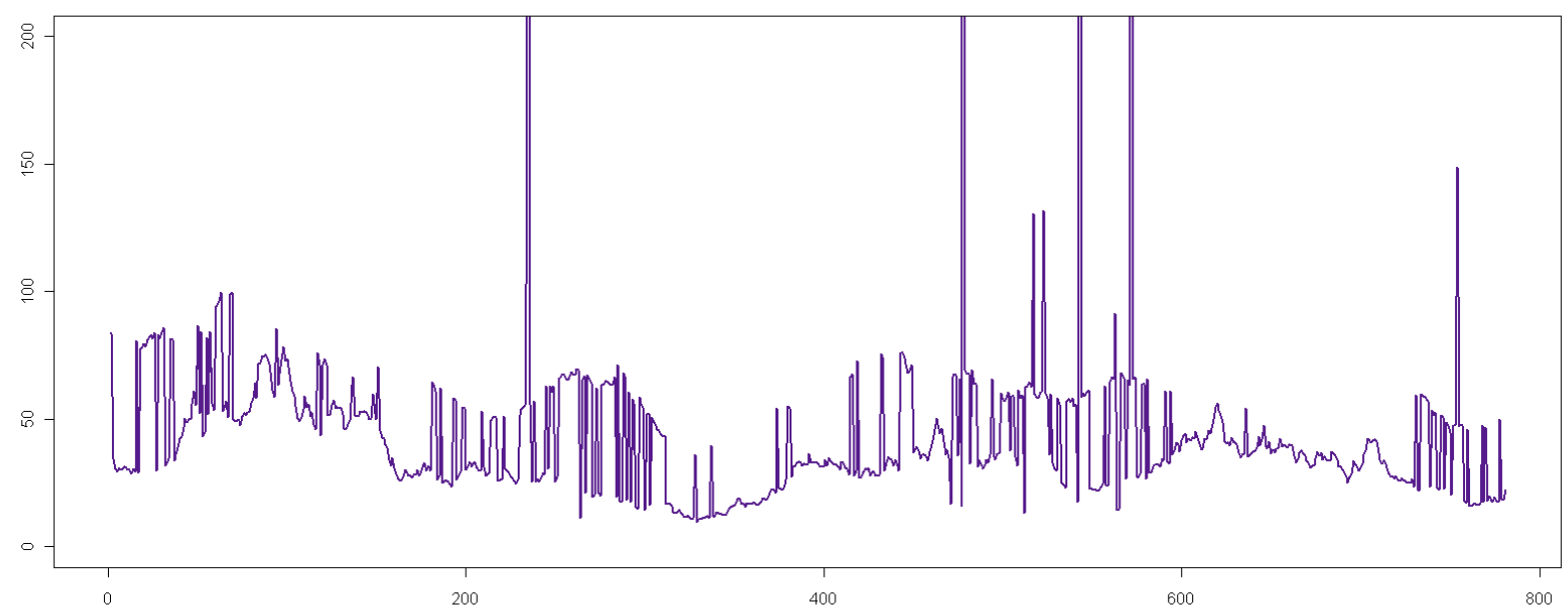

Figure 6: Spot price simulation. Parameters calibrated on the period 01/2007 - 12/2008. We use two thresholds for very high price peaks (when $D_{t}>8500 \mathrm{MWh}$, the price is fixed to $500 €$ ) and low demand prices (when $D_{t}<0 \mathrm{MWh}$, the price is fixed to $15 €$ ). The process is simulated on 780 points (3 years).

[3] J. Batut and A. Renaud, Daily generation scheduling with transmission constraints: A new class of algorithms, IEEE Transactions on Power Systems 7 (1992), 982-989.

[4] F. E. Benth, J. S. Benth, and S. Koekebakker, Stochastic modeling of electricity and related markets, World Scientific Publishing Company, 2008.

[5] F. E. Benth, L. Ekeland, R. Hauge, and B. F. Nielsen, A note on arbitrage-free pricing of forward contracts in energy markets, Applied Mathematical Finance 10 (2003), no. 4, 325-336.

[6] F. E. Benth and S. Koekebakker, Stochastic modeling of financial electricity contracts, Journal of Energy Economics 30 (2007), 1116-1157.

[7] F. E. Benth, S. Koekebakker, and F. Ollmar, Extracting and applying smooth forward curves from average-based commodity contracts with seasonal variation, Journal of Derivatives 15 (2007), no. 1, $52-66$.

[8] T. Björk, Arbitrage theory in continuous time, Oxford University Press, 2004.

[9] M. Burger, B. Graeber, and Gero Schindlmayr, Managing energy risk: An integrated view on power and other energy markets, Wiley, 2008.

[10] M. Burger, B. Klar, A. Müller, and G. Schlindmayr, A spot market model for pricing derivatives in electricity markets, Quantitative Finance 4 (2004), 109-122.

[11] A. Cartea and M.G. Figueroa, Pricing in electricity markets: a mean reverting jump diffusion model with seasonality, Applied Mathematical Finance 12 (05), no. 4, 313-335.

[12] L. Clewlow and C. Strickland, Energy derivatives, Lacima Group, 2000.

[13] F. Delbaen and W. Schachermayer, A general version of the fundamental theorem of asset pricing, Mathematische Annalen 312 (1994), 215-250. 
[14] D. Dentcheva, R. Gollmer, A. Möller, W. Römisch, and R. Schuttz, Solving the unit commitment problem in power generation with primal and dual methods, Progress in Industrial Mathematics (Teubner, Stuttgart) (M. Brøns, M.P. Bendsøe, and M.P. Sørensen, eds.), ECMI 96, 1997, pp. 332-339.

[15] A. Eydeland and K. Wolyniec, Energy and power risk management: New developments in modeling, pricing and hedging, Wiley, 2002.

[16] S.-E. Fleten and Lemming J., Constructing forward price curves in electricity markets, Energy Economics 25 (2003), 409-424.

[17] H. Geman, Commodities and commodity derivatives: Modelling and pricing for agriculturals, metals and energy, Wiley, 2007.

[18] H. Geman and A. Roncoroni, Understanding the fine structure of electricity prices, Journal of Business 79 (2002), no. 3, 1225-1262.

[19] Föllmer H. and M. Schweizer, Hedging of contingent claims under incomplete information, Applied stochastic analysis (London, 1989), Stochastics Monogr., 5, Gordon and Breach, New York (1991), $389-414$.

[20] I. Karatzas, Lectures on the mathematics of finance, CRM Monograph Series, 8. American Mathematical Society, Providence, RI, 1997.

[21] I. Karatzas and S. E. Shreve, Brownian motion and stochastic calculus, Springer-Verlag, 1991.

[22] B. R. Routledge, D. J. Seppi, and C. S. Spatt, Equilibrium forward curves for commodities, The Journal of Finance $5 \mathbf{5}$ (2000), no. 3, 1297-1338.

[23] E. Schwartz, The stochastic behavior of commodity prices: Implications for valuation and hedging, The Journal of Finance 52 (1997), no. 3, 923-973.

[24] M. Schweizer, A guided tour through quadratic hedging approaches, Option pricing, interest rates and risk management, Handb. Math. Finance, Cambridge Univ. Press, Cambridge (2001), 538-574. 